\title{
ENTREVISTA \\ EL GRUPO DE INVESTIGACIÓN DEMENCIA 10/66
}

\author{
Adolfo Israel Flores Ramírez \\ Posgrado en Filosofía de la CienciaUNAM \\ polemospater@hotmail.com
}

\section{DOI: https://doi.org/10.38128/cienciayfilosofa.v2i2.13}

Sólo el $10 \%$ de todas las investigaciones sobre demencias se realizan en los países en vías de desarrollo, donde vive aproximadamente el $66 \%$ de todas las personas con demencia, de ahí el nombre del grupo de investigación Demencia 10/66. El grupo está formado por investigadores, que trabajan en países de bajos y medianos ingresos y es coordinado por el profesor Martín Prince, del Institute of Psychiatry, De Crespigny Park, en Londres. El grupo se formó en 1996 para subsanar la escasez de investigaciones en Demencia en los países subdesarrollados.

La representante en México del grupo de investigación Demencia 10/66 es la Doctora Ana Luisa Sosa Ortiz. En las siguientes líneas reproducimos la entrevista que nos concedió en su oficina el 4 de junio de 2012 en la Ciudad de México.

La Dra. Ana Luisa nos explicó que la investigación sobre Demencia abarca diversas esferas:

"Nosotros trabajamos, desde lo social hasta lo molecular, trabajamos con pacientes de la clínica de demencias, los atendemos, les hacemos diagnostico, les damos tratamiento, les ofrecemos alternativas, le damos educación a la familia porque cuando hay un problema de demencia, no sólo afecta a la persona que la padece, sino que hay un binomio inseparable que es el paciente y el cuidador, además, uno de los problemas de la demencia es que ocasiona un alto costo socioeconómico porque ocasiona discapacidad. Y es la principal causa de discapacidad en el adulto mayor, entonces, si por ejemplo tú tuvieras a un abuelo o un familiar, con discapacidad, con demencia, ya no se puede quedar sólo, entonces alguien tiene que cuidarlo, la familia tiene que buscar soluciones para el cuidado de esa persona dependiente ¿Cuáles pueden ser las soluciones? Pues que alguien [de la familia] deje de trabajar, se quede a cuidar, y puede haber un ingreso menos o bien que un miembro joven de la familia se quede a cuidarlo o bien que paguen a alguien para cuidarlo. Entonces esto va a causar un mayor empobrecimiento de las familias que tienen este tipo de problemas. 
No contamos con programas especiales [En México] para atender este tipo de Población. No hay un programa medico que fuera capaz por sí mismo de resolver esta problemática creciente. Es un problema cuyo principal riesgo es en adultos mayores.

Todos nacemos con un capital de neuronas que diariamente se nos van gastando y muriendo, entonces ¿quién ha perdido más neuronas? Pues el que tiene más años, entonces ¿a quiénes les va a dar demencia? Pues a las personas que nacieron con una pobre dotación, o que no consiguieron mantener en la vida una buena reserva neuronal o que tuvieron pérdidas adicionales de neuronas por diferentes motivos, pueden ser desde un golpe, un traumatismo craneoencefálico que se mueren muchas neuronas o problemas de intoxicación, por ejemplo por el alcohol, por problemas deficitarios, por falta de alimentación, mala nutrición. Hay nutrientes que son indispensables para el buen funcionamiento del cerebro.

Hay más de 60 causas de Demencia, una de ellas es la enfermedad de Alzheimer, entonces, sabemos que la población mundial está envejeciendo y México no es la excepción, nada más que los países en desarrollo a diferencia de los países ya desarrollados, pues envejecimos rápidamente. Los países desarrollados envejecieron lentamente mientras se enriquecían y pues a nosotros ya no nos dio tiempo, entonces estamos con problemas, todavía en la transición demográfica, que es el crecimiento de la población envejecida, donde el número de nacimientos ha disminuido entonces, la población ha envejecido, llegará un momento en que va a llegar a igualar o superar a la población joven, y es una población que se caracteriza por tener más factores de riesgo y por ser dependiente.

Los países del mundo no han podido solucionar [el problema de la demencia] con un programa médico, tiene que haber programas sociales, sanitarios que nos involucre a todos, para poder dar respuesta e este tipo de variables.

Nosotros hemos estado trabajando la frecuencia de demencias en México, este estudio es el primero, que hace una encuesta buscando precisamente la presencia de demencia. Participamos con el grupo Demencia 10/66, nos reunimos varios países que no teníamos información epidemiológica en nuestros países, entonces, utilizábamos los datos de otros países. [inferíamos que] Si Estados Unidos tiene tantos casos de demencia, nosotros tenemos tantos casos de demencia.

Tenemos un estudio que se hizo en áreas determinadas, con mexicanos, un área urbana, un área rural, y sabemos que aproximadamente $7.5 \%$ de los adultos mayores de 60 años pueden tener demencia. Eso es un numero que está arriba de los 600000 individuos, si esto lo multiplicas por dos, por el cuidador involucrado, están siendo afectados directa o indirectamente por este problema más de un millón de mexicanos. 
¿A esto le llaman la sobre carga del cuidador?

La sobre carga del cuidador es cuando el cuidador ya llegó a su límite, está colapsando porque cuando tu le enseñas a un niño, sabes que le vas a ir enseñando cosas y el va a ir de la mano haciéndose cada vez más independiente, entonces eso te va dando el estímulo ¿no? Porque con el tiempo eso va a mejorar, en cambio con el adulto mayor, lamentablemente cada vez va a ser peor, porque es un padecimiento progresivo, primero tienes que estar al pendiente, después lo tienes que supervisar, hacer todas las tareas y después tienes que hacer todo por él. Entonces el paciente olvida hasta caminar, ir al baño, todo, entonces es alguien que depende de otro y esa dependencia es cada vez mayor.

¿Qué es lo que tiene que saber una persona que se encuentra, digamos en Iztapalapa, donde el nivel cultural de la gente es muy bajo, y no tiene tiempo de leer una gaceta para enterarse de estas cosas? ¿Qué sería lo mínimo que ellos tendrían que saber para identificar la Demencia? porque muchas veces se da el caso, y, a los adultos mayores los tachan de testarudos sin darse cuenta de que hay problemas. Si iniciamos una campaña ¿qué le podemos decir a la población?

Yo les daría tres mensajes: uno, que hay que diferenciar entre el envejecimiento normal, o sea lo que les pasa a todas las personas cuando envejecen y lo que es una enfermedad. Es usual que las personas con la edad vayan perdiendo capacidades físicas y mentales, pero no porque alguien suba más lento las escaleras está enfermo, pero alguien que no puede caminar sólo pues sí está enfermo. Lo mismo pasa en las demencias, es normal que las personas con la edad tengamos menos velocidad de procesamiento, de aprendizaje para entender las cosas y podamos tener algunos olvidos. Que también está muy mediado por problemas en la atención, entonces a veces es muy típico, a veces la gente se queja de que va a hacer una cosa y en el camino se le olvidó a qué iba ¿a qué venía a la cocina? o que se encontró algo en el camino, a lo mejor encontró algo tirado lo levantó, ya no sabe ni a qué iba. Eso es la atención dividida, disminuye con la edad. Si a mí me dan ahorita algo para aprender, me tardo más que hace 20 años y a la mejor tardo más tiempo en procesarlo pero tengo otros beneficios como puede ser el conocimiento acumulado, la experiencia, la prudencia, en fin. Entonces, yo puedo tener por mi edad un enlentecimiento y un cambio en mis funciones mentales. Pero ese cambio no debe interferir con mi funcionalidad, con mi independencia. Entonces hay que diferenciar cuando una persona tiene cambios que se están atribuyendo al enlentecimiento pero esto lo hace disfuncional y dependiente eso ya es una enfermedad. Mientras este cambio es lento y puede afectar simplemente mi velocidad pero no mi autonomía, puede considerarse normal. Si no sucede eso, se considera una enfermedad y en el caso de las demencias necesitamos evidenciar dos situaciones: una que hay un deterioro del funcionamiento 
mental, el más frecuente es el de la memoria y dos, que es el deterioro del funcionamiento mental y aparte mi funcionalidad ¿qué quiere decir esto? Que yo no pueda hacer las cosas que yo solía hacer ni fuera de mi casa ni dentro de mi casa. Que yo no me pueda bañar, no me pueda vestir, no pueda preparar mis alimentos, no pueda ir al super, no pueda salir de mi casa porque me pierdo. Eso sería el primer mensaje, la diferencia entre envejecimiento normal y demencia.

Lo segundo, una vez que se instala la demencia, no se cura, se puede frenar sólo su evolución. Algunos casos se trabaja la calidad de vida del paciente y de su familiar ¿qué quiere decir esto? Que yo voy a buscar que mi paciente esté bien cuidado, que duerma bien, que no se angustie.

El tercer mensaje sería que lo mejor es prevenir, si hay factores de riesgo para la demencia como puede ser la hipertensión, la diabetes, el colesterol, los traumatismos craneoencefálicos, el exceso de peso, la vida sedentaria, entonces yo voy a tratar de combatir esos factores de riesgo, que sé que van a facilitar la demencia, entonces, hay que tratar de evitar los excesos como fumar, beber, tener una vida saludable y una buena alimentación. Ese sería mi consejo.

¿De qué manera puede el proyecto Demencia 10/66 llegar a esferas amplias de la sociedad?

Estamos trabajando en varios lugares. Trabajamos en un modelo de centro de día, para plantear qué es lo que se necesita y qué es lo que se debe hacer. Un centro de día modelo para pacientes con demencia. Esto quiere decir, si yo tengo a mi abuelita con Alzheimer, en vez de quedarme sin trabajar, en vez de que mi hijo no vaya a la escuela, en vez de pagar una enfermera, la llevo a un centro donde no sólo la van a cuidar, sino la van a estimular para que sea funcional por más tiempo y los costos de este tipo de lugares, es simbólico, de acuerdo a las posibilidades económicas de cada quien. Son instituciones no lucrativas. Esa es una opción, otra opción que estamos trabajando junto con el instituto nacional de pediatría una propuesta para un plan nacional de demencias, esto es, para que haya detección y educación. Detección de los pacientes y educación, tanto para el médico general, personal de salud y población en general.

La Doctora Ana Luisa Sosa es responsable del Laboratorio de Demencia del Instituto Nacional de Neurología y Neurocirugía "Manuel Velazco Suarez", colabora en el grupo de Investigación Demencia 10/66, ha publicado numerosos artículos sobre la Demencia en ancianos y colabora con la organización Alzheimer Disease international. Entre los trabajos publicados por la Dra. Sosa, se encuentran los estudios sobre Demencia en adultos mayores en Latinoamérica y la relación entre los pacientes con Demencia y sus familias. La Organización Mundial de la Salud y la organización 
Alzheimer Disease International, publicaron este año (2012) el informe titulado Demencia una prioridad de salud pública. 